\title{
DISTRIBUZIONE STATISTICA DELLE PARTICELLE $\alpha$ RACCOLTE SU UNA LASTRA FOTOGRAFICA IN FUNZIONE DELLA PROIEZIONE DEL PERCORSO NELLL'EMULSIONE
}

\author{
G. Imiò - L. Casentano
}

1. - Per lo studio della radioattività delle lave vesuviane conviene seguire il metodo delle lastre fotografiche con emulsioni nucleari, particolarmente per la debole attivita del materiale.

In ricerche analoghe ( $\left.{ }^{1}\right)$, per il calcolo del rapporto in peso della concentrazione del torio $\left(C_{\mathrm{rh}}\right)$ rispetto a quella dell'uranio $\left(C_{v}\right)$ è stato applicato il metorlo di Curie ( $\left.{ }^{2}\right)$, loasato sul fatto che nell'emulsione le tracee aventi una lunghezza maggiore a un percorso equivalente nell'aria di $\mathrm{cm} 7$ sono dovute esclusivamente a particelle $\alpha$ derivanti dal $T h C^{\prime}$, e quelle invece con un percorso equivalente nell'aria compreso fra $\mathrm{em} \quad \tau$ e $\mathrm{cm} 5.8$ sino tovute al particelle a del $T h C^{\prime}$, del $R a C^{\prime}$ e dell'AcA.

II rapporto considerato si ricava da una formula stalilita dalla Curic $(-1)$ c che può mettersi anche sotto la seguente forma:

$$
\frac{C_{\mathrm{v}}}{C_{\mathrm{Th}}}=0.30 \frac{n_{1}}{n_{\mathrm{g}}}-0.24
$$

dove $n_{1}$ e $n_{2}$ sono il numero di tracce, contate sulla stessa superficie, con percorso equivalente nell'aria rispettivamente maggiore di $\mathrm{cm} 7$ e compreso fra $\mathrm{cm} 7 \mathrm{e} \mathrm{cm} 5.8$.

Tenendo presente la difficoltà (e l'impossililità per le particelle che entrano nell'emulsione in direzione normale alla lastra) di misurare la lunghezza ellettiva del percorso - per la qual cosa la Curie stessa attribuisce ai risultati sirnificato soltanto indicativo - si è voluto ricorrere ad altro metodo, usando le proiezioni dei percorsi, nell'emulsione, delle particelle a.

Per poter fare ciò era necessario conoscere la distribuzione statistica delle particelle $u$ racolte su una lastra fotografica in fumzione appunto delle proiezioni sul piano della lastra del percorso nell'emulsione. 
In questo primo studio ei proponiamo di trovare tale distribuzione, fermandoci a considerare l'emissione di particelle a dalle tre famiglie separatamente. In seguilo si dari conto del metodo che si vorrà seguire per la soluzione del problema proposto, del quale metodo però se ne potrì, nelle linec generali, prevedere lo sviluppo già da quanto si andrà dicendo adesso.

2. - Si abbia una sostanza radioatliva, emittente un solo tipo di particelle $\alpha$, a contatto con la lastra fotografica con emulsione speciale. Dalla sostanza si ha l'emissione di particelle in tutte le direzioni.

Considerando nullo l'eventuale effetto sensibile di rifrazione per il passaggio di una particella u dalla sostanza radioattiva nell'emulsione, uguale cioè la sua velociti nei due mezzi, la proiezione del cammino nell'emulsione di tale particella $a$, emessa da $A$ in direzione $f F$, sarì data da $G E$ (v. firg. 1 ).

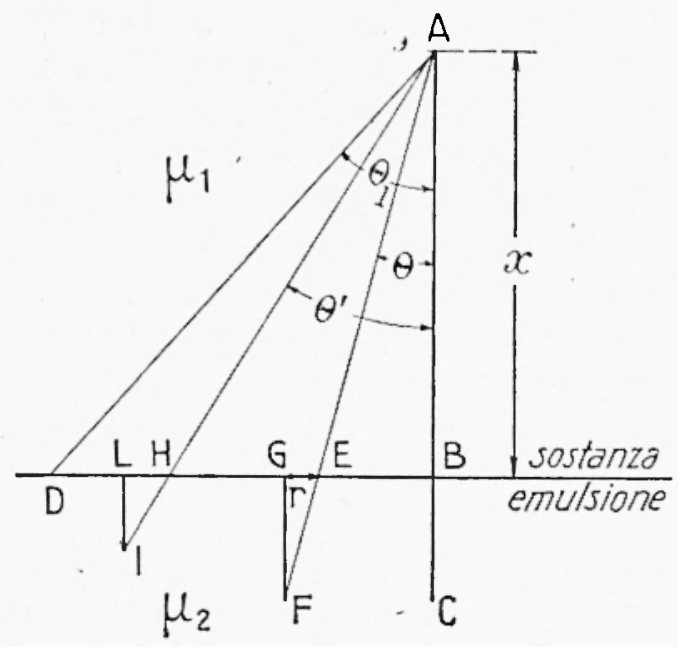

Fig. 1

Indicando con $R$ il range nell'aria, e con $R_{1}$ e $R_{2}$ rispettivamente quello nella sostanza e nell'emulsione, con $\mu_{1}=\frac{R_{1}}{R}$ e $\mu_{2}-\frac{R_{2}}{R}$ il coefficiente di frenamento, inverso del potere di arresto, della sostanza e dell'emulsione, la proiezione $(G E=r$ ) in funzione della distanza $x$ dall'emulsione di 4 e dell'angolo $v$ che la direzione di emissione forma con la normale alla superficie della lastra, considerando che $i$ :

$$
\frac{A E}{\mu_{1}}+\frac{F F}{\mu_{2}}=R \text {, }
$$


è lata da:

$$
\left.\frac{r}{\mu_{2}}=R \operatorname{sen} i-\frac{x}{\mu_{1}} \operatorname{tag}\right\}
$$

La soluzione della [1] rispetto a $\}$, che è il parametro che interessa per risolvere il nostro problema analogamente a quanto fatto da Evans (") per le lunghezze effettive dei percorsi, non presenta difficolta analitiche insormontahili; ma l'espressione che si ricara non i tale che possa permettere in modo relativamente semplice e preciso la ulteriore conduzione dei calcoli.

Per lale ragrione si i preferito sceglierc tutt'altra strada.

3. - Si è considerato che delle particelle $u$, emesse da un elemento di rolume in $A$, hanno la proiezione prima definita ridotta a un punto, sia quelle dirette secondo $A B$ (in modo che $\dot{e} \cos i=1$ ) sia quelle dirette lungo la semiretta $A D$, per le quali ̀̀ $A D=R_{1}$ (in modo che $i \cos i)=\frac{x}{u_{1} k}$ : valore limite dell'angolo che si indica con $i_{1}$ ). Per ) variabile nei campi determinati da:

$$
0 \leqslant 0 \leqslant \eta_{m} \text { e } \quad \eta_{1} \geqslant i \geqslant 0 \text { m }
$$

dove $n_{m}$ si ricava da:

$$
\left.\cos ^{0}\right)_{\ldots}=\left(\frac{x}{\mu_{1} R}\right)^{1 / 3}
$$

$r$ varia da $i$ fino ad un valore massimo $r_{m}$, corrispondente all'angolo di cmissione $\eta_{n}$, e dato da:

$$
r_{\mathrm{m}}==! !_{\mathrm{n}} R\left[1-\left(\frac{x}{l_{1} R}\right)^{2 / 3}\right]^{3 / 2}
$$

Quindi del totale delle particelle $\alpha$, emesse dal cubetto elementare considerato, il numero delle particelle aventi la proiezione del percorso nellemulsione compresa fra $O$ e un valore $r<r_{\mathrm{m}}$ ¿̀ dato dalla somma dei numeri di quelle arenti direzioni di propagazione comprese:

a) nell'angolo solido determinato dalla superficie conica avente cone asse la semirelta $A B$ e come generatrice la semiretta $A F$ rappresentante la direzione di emissione che dà $G E=r$;

b) nell'angolo solido $\Omega_{1}=\Omega-\Omega^{\prime}$ con $\Omega$ e $\Omega^{\prime}$ determinati dalle due superfici coniche aventi come asse sempre la semiretla $A B$ e come generatrici rispcttiramente la semiretta $A D$ e l'altra $A I$. che rap- 
presentano, la prima la direzione limite di cui già si è parlato, e, la seconda, la direzione di emissione, con $\eta>\eta_{m}$, per cui si ha ancora $L H=r$.

Poiché il numero delle particelle $x$ emesse in un angolo solido è proporzionale al valore dell'angolo solido stesso, ¿̀ possibile trovare la distribuzione statistica che ci interessa per le particelle a emesse da uno strato di sostanza radioattiva, di spessore infinitamente sottile, posto alla distanza $x$.

L'unica difficolta che si incontra, e cioè la mancata conoscenza del valore del segmento $L I$, vicne superata volta per volta graficamente.

Per seguire il metodo ideato da uno di noi (Casertano), si è innanzitutto calcolata la distribuzione per due strati infinitamente sottili posti rispettivamente alle distanze limiti per cui si ha $\frac{x_{1}}{\mu_{1}}=O$ e $\frac{x_{1}}{\mu_{2}}=R$. (Il contributo del secondo viene considerato in linea esclusivamente teorica, essendo in effetti nullo).

derato in linea esclusivamente teorico, essendo in effetti nullo).

É stata poi considerata la distribuzione che si ottiene con l'interporre un terzo strato alla distanza che dà $\frac{x_{*}}{\mu_{1}}=\frac{1}{2} R$, c così quella complessiva ottenuta via via che si infittiscono gli strati con opportuni criteri.

La distribuzione cercata per una sostanza di spessore $x>R_{1}$ non può che essere quella che si oitiene per un numero infinito di strati sottili, dei quali i limiti sono i primi due strati considerati.

Per trovare la distribuzione per infiniti strati sottili si sono infittiti gli strati fino ad arrivare al numero di 161. Nei grafici delle fig. 2 e fig. 3 sono riportati $i$ valori ottenuti per 11,2$], 41,81$, e 161 strati.

Nella fig. 2 le ordinate, indicate con $n$, rappresentano il percento delle particelle $\alpha$ aventi la proiezione nell'emulsione comprese fra $O$ e il valore dell'ascissa corrispondente. Nella fig 3 , invece, si indicano con $\Delta_{n}$, e rappresentano il numero di particelle $a$, sempre su un totale di cento, con proiczione corrispondente al valore dell'ascissa.

Si vede nella figr. 2 che la distribuzione per 161 strati può considerarsi cone limite, e che essa non viene a essere che impercettibilmente modificata quando nella figr. 3 si prende come linite quella 
piì marcata. Quest'ultima si è ottenuta molto facilmente tenendo conto delle variazioni che vanno man mano subendo quelle derivanti da un numero sempre maggiore di strati.

I valori numerici delle due curve limiti, sono riportati nella tabella $l$.

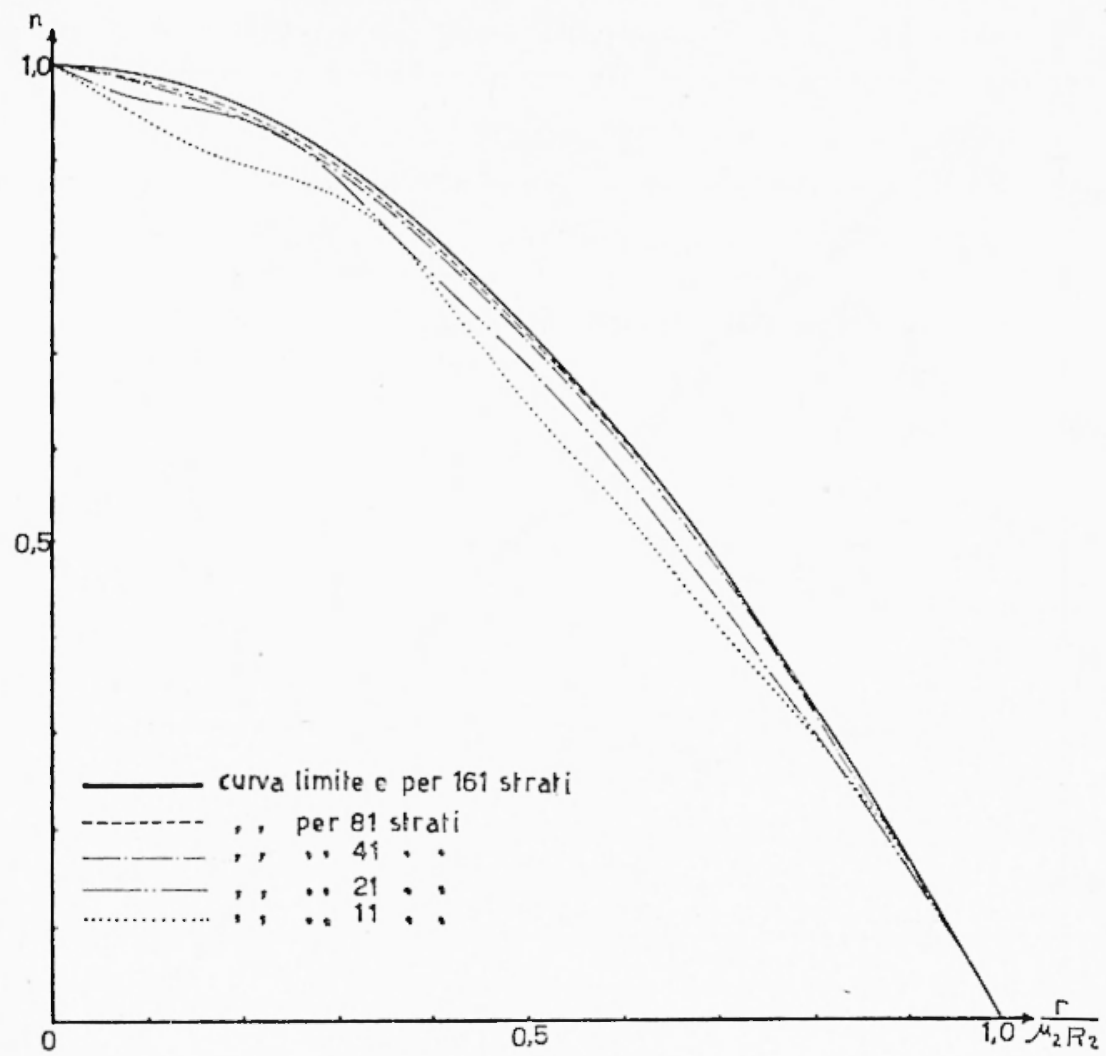

Fig. 2

1. - Nel paragrafo precedente è stata studiata la distribuzione che si ottiene da una sostanza emittente un solo tipo di particelle $"$. Si vuole adesso csaminare il caso di una famiglia con tutti gli elementi in equilibrio radioattivo.

Poiché in tale condizione i singoli elementi emettono, nell'unità di tempo, lo stesso numero di particelle, e ognuno contribuisce, al totale raccolto su una lastra fotografica, con uno spessore di sostanza uruale al range nella sostanza in esame, possono facilmente ricavarsi i contrihuti dei vari elementi. 
Supponendo raccolte, per ognuna delle tre famiglie scparatamentc, 100 particelle $x$, il numero di quelle derivanti dai singoli elcmenti delle famiglie i riportato nella tabella 11.

In base ai risultati del paragrafo precedente per ogni membro di una famiglia può trovarsi la distribuzione delle parlicelle a rac-

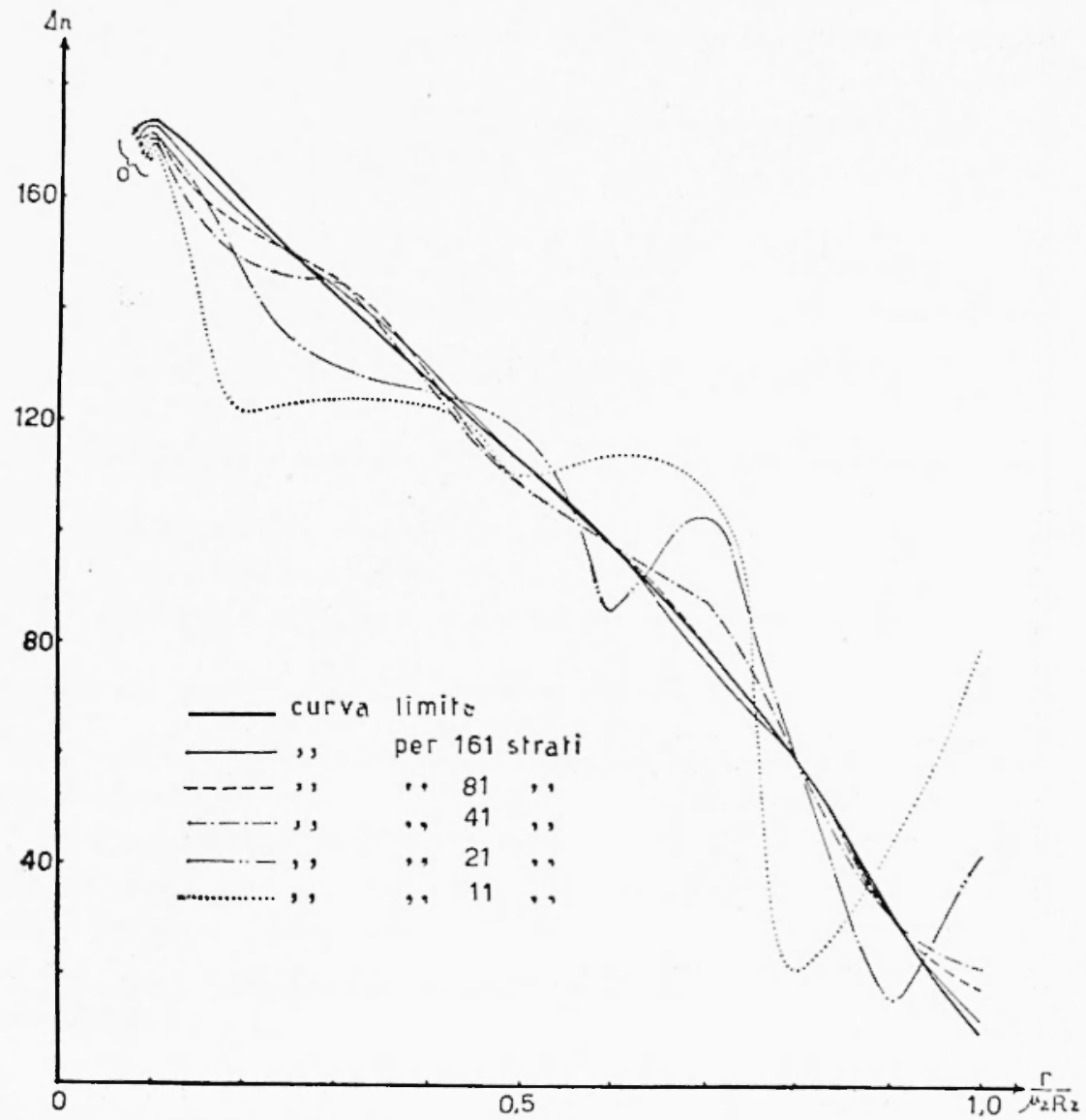

Fig. 3

colte in funzione alel percorso nell'emulsione, e quindi la distribuzione complessiva per ogni famiglia. indipendentenente l'una dallaltra.

Nella tahella III sono riportati i valori ottenuti dai calcoli, c. riprodotli nei grafici delle fig. 4 e fig. 5 .

5. - Abluiamo ottenuto cosi la distribuzione statistica percentuale 


\section{Tabelita I}

\begin{tabular}{|r|r|r|r|r|r|r|r|r|r|r|}
\hline $\mathbf{r}$ & 0.1 & 0.2 & 0.3 & 0.1 & 0.5 & 0.6 & 0.7 & 0.8 & 0.9 & 1.0 \\
\hline$\mu_{3} \mathbf{R}_{3}$ & & & & & & & \\
\hline $\mathrm{n}$ & 17.1 & 33.4 & 17.7 & 601.5 & 71.8 & 81.5 & 89.5 & 95.4 & 98.9 & 100.0 \\
\hline $\mathrm{sn}$ & 17.1 & 16.0 & 11.3 & 12.8 & 11.3 & 9.7 & 8.0 & 5.9 & 3.5 & 1.1 \\
\hline
\end{tabular}

TabelLa II

\begin{tabular}{|c|c|c|c|c|c|}
\hline \multicolumn{2}{|c|}{ IRANIO } & \multicolumn{2}{|c|}{ ATTINIO } & \multicolumn{2}{|c|}{ TORIO } \\
\hline Elemento & Niumero & Elemento & Numero & Elemento & Numero \\
\hline $\mathrm{U}_{1}$ & 8.1 & ACU & 8.6 & $\mathrm{Th}_{3}$ & 9.1 \\
\hline III & 10.1 & $\mathrm{P}_{\mathrm{il}}$ & 10.6 & RTlı & 13.8 \\
\hline lo & 9.8 & RAc & $1+1.3$ & $\mathrm{ThX}$ & 14.9 \\
\hline Ra & 10.3 & ACX & 13.1 & $T_{n}$ & 17.4 \\
\hline$R_{n}$ & 12.8 & $A_{n}$ & 17.3 & ThA & 19.6 \\
\hline Rat & 1.1 .7 & A.A & 19.6 & $\mathrm{Th} \mathrm{C}$ & 5.8 \\
\hline Rac:' & 21.8 & $\mathrm{ACC}$ & 16.5 & $\mathrm{Th}_{1} \mathrm{C}^{\prime}$ & 19.4 \\
\hline Po & 12.1 & & & & \\
\hline Totile & 100.0 & Totale & 100.0 & Totiale & 100.0 \\
\hline
\end{tabular}

TaBFitaA III

\begin{tabular}{|c|c|c|c|c|c|c|c|c|}
\hline \multicolumn{3}{|c|}{ ERIVIO } & \multicolumn{3}{|c|}{ ATTINIO } & \multicolumn{3}{|c|}{ TORIO } \\
\hline$r$ in $r m$ & $n$ in & $\ln _{\mathrm{n}}$ in & $\mathrm{r}$ in $\mathrm{cm}$ & $n$ in $r_{c}^{\prime}$ & In in $\%$ & $\mathrm{r}$ in $\mathrm{cm}$ & $n$ in $\%$ & In in 90 \\
\hline 0.60 & 29.3 & 29.3 & 0.65 & 23.8 & 23.8 & 0.86 & 29.8 & 29.8 \\
\hline 1.38 & 53.6 & 2.1 .3 & 1.29 & 43.9 & 20.1 & 1.71 & 53.0 & 23.2 \\
\hline 2.117 & $i 2.7$ & 19.1 & 1.9 .4 & 01.2 & 17.3 & 2.57 & 72.7 & 19.7 \\
\hline 2.70 & 85.4 & 12.7 & 2.58 & 71.3 & 13.6 & 3.13 & 85.5 & 12.8 \\
\hline 3.45 & (9. .2 & 6.8 & 3.23 & 85.0 & 110.2 & 4.29 & 92.7 & 7.2 \\
\hline 4.11 & 95.7 & 3.5 & 3.87 & 92.1 & 7.1 & 5.14 & 96.2 & 3.5 \\
\hline 1.83 & 97.7 & 2.0 & 4.52 & 96.5 & 4.4 & 6.00 & 98.0 & 1.8 \\
\hline 5.53 & 99.11 & 1.3 & 5.17 & 98.8 & 2.3 & 6.86 & 99.1 & 1.1 \\
\hline 6.22 & 99.8 & 0.8 & 5.81 & 99.8 & 1.0 & 7.71 & 99.8 & 0.7 \\
\hline 6.91 & 100.0 & 0.2 & 6.16 & 100.0 & 0.2 & 8.57 & 100.0 & 0.2 \\
\hline
\end{tabular}

Le proiezioni nellemulsione sono espresse in percorsi equivalenti in $\mathrm{cm}$ nellaria; per ogni famiglia si $i$ scelto un intervallo corrispondente a $1 / 10$ del più lungo range della famiglia stessa. 


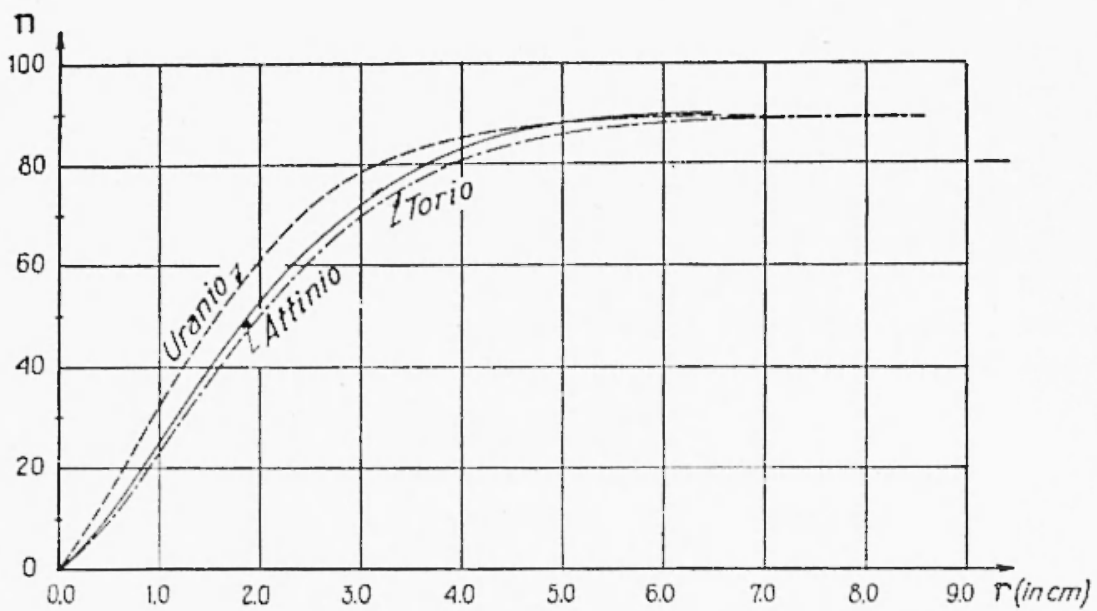

Fig. 4

delle particelle a raccolte su una lastra per ricerche radioattive, quando le particelle sono emesse:

a) da una sola specie di nucleo radioattivo;

b) dagli elementi di una sola famiglia in equilibrio radioattivo.

Si nota facilmente che il metodo non è esclusivo per il tipo di ricerche esaminato, e che può esscre applicato anche con altri contatori che permettano la misura della proiezione del pereorso nell'interno del contatore di ogni singola particella u.

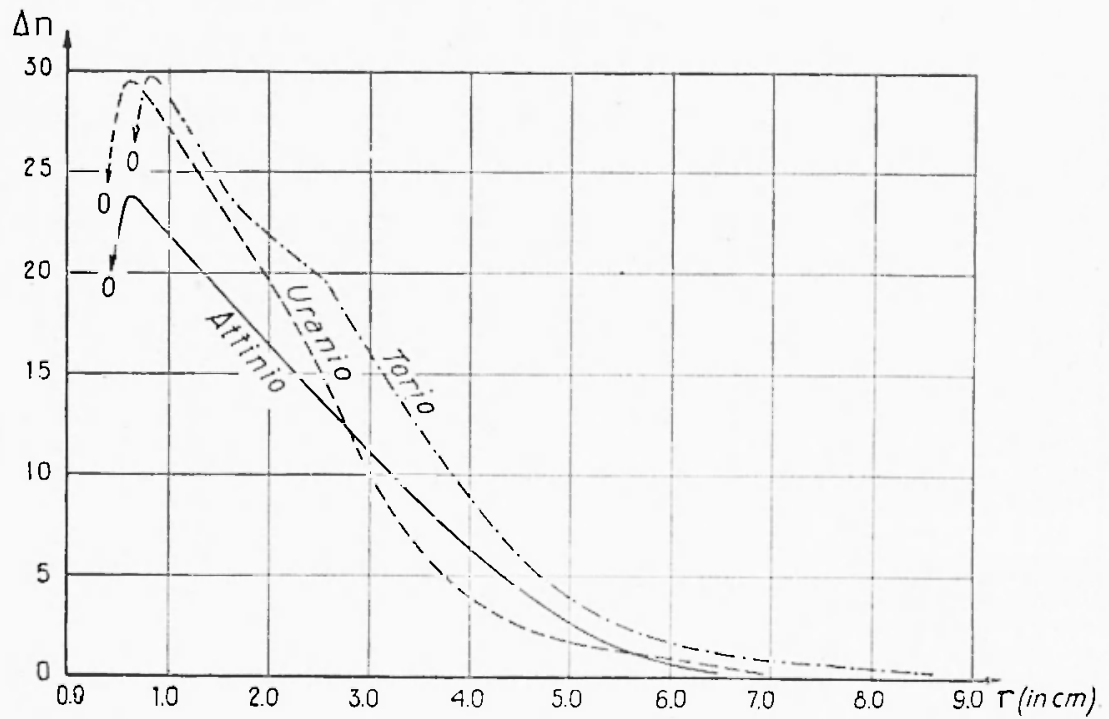

Fig. 5 
Successivamente si farà vedere come dalla distribuzione statistica ottenuta sperimentalmente per particelle $\alpha$ provenienti da una sostanza contenente gli elementi delle tre famiglie si può risalire non solo al valore del rapporto $C_{\mathrm{rr}} / C_{\mathrm{r}}$, ma anche a quello della percentuale di questi due clementi.

Napoli - Istituto di fisica terrestre dell'Università-Ottobre 1950.

\section{RIASSUNTO}

Allo scopo di poter successivamente determinare il tenore dei singoli elementi radioattivi nelle rocce, si deducono le curve di distribuzione delle particelle a, raccolte su una lastra fotografica con emulsione speciale per ricerche radioattive, in funzione della proiezione orizzontale del percorso delle particelle nell'emulsione.

Oltre allo studio per elementi che emettono un solo tipo di particelle "s sono prese in considerazione anche distribuzioni relative alle singole famiglie radioattive in equilibrio.

\section{BIBIJIOGRAFIA}

(1) Coppess, Journal de Phys. XI-21-1950. In questo articolo l'autore dà conto di varie note publ,licate la maggior parte sui «Comptes Rendus».

Pucciotro, Bull. Soc. belge de Géol. LVIII-76-1949.

(-) J. Cunte, Journal de Phys. VII-313-1946.

(3) Exass, Physical Rev. 45-25.1934. 